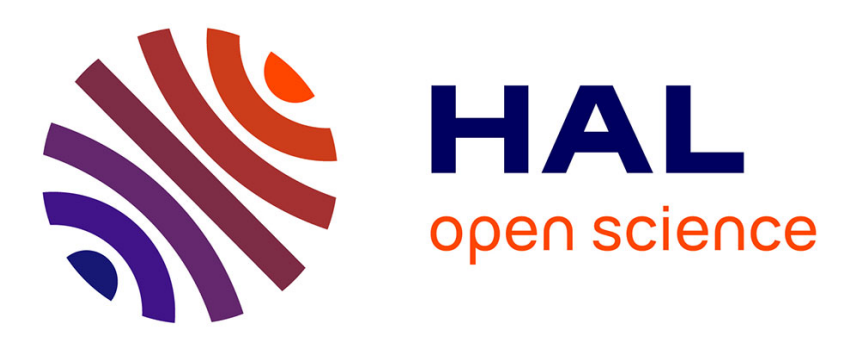

\title{
Boundary Control Design for Linear Conservation Laws in the Presence of Energy-Bounded Measurement Noise
} Francesco Ferrante, Christophe Prieur

\section{To cite this version:}

Francesco Ferrante, Christophe Prieur. Boundary Control Design for Linear Conservation Laws in the Presence of Energy-Bounded Measurement Noise. CDC 2018 - 57th IEEE Conference on Decision and Control, Dec 2018, Miami, FL, United States. pp.6550-6555. hal-01880804

\section{HAL Id: hal-01880804 https://hal.science/hal-01880804}

Submitted on 20 Dec 2021

HAL is a multi-disciplinary open access archive for the deposit and dissemination of scientific research documents, whether they are published or not. The documents may come from teaching and research institutions in France or abroad, or from public or private research centers.
L'archive ouverte pluridisciplinaire $\mathbf{H A L}$, est destinée au dépôt et à la diffusion de documents scientifiques de niveau recherche, publiés ou non, émanant des établissements d'enseignement et de recherche français ou étrangers, des laboratoires publics ou privés. 


\title{
Boundary Control Design for Linear Conservation Laws in the Presence of Energy-Bounded Measurement Noise
}

\author{
Francesco Ferrante and Christophe Prieur
}

\begin{abstract}
Boundary feedback control design for a system of $n$ 1-D linear conservation laws is studied. Sufficient conditions in the form of Lyapunov-like functional inequalities are given to certify the existence of a bound on the $\mathcal{L}^{2}$ (spatial) norm of the state with respect to energy bounded measurement noise. Semidefinite programming techniques are adopted to devise a systematic design algorithm. The effectiveness of the approach is shown in a numerical example.
\end{abstract}

\section{INTRODUCTION}

The tight description of many physical phenomena relies on mathematical models in which variables that depend simultaneously on space and time are related each other through differential relationships. This leads to systems modeled by partial differential equations (PDEs). Systems modeled via PDEs are omnipresent in physical sciences. For example, PDEs play a central role in: transport phenomena in fluid mechanics, heat diffusion in thermic systems, vibratory behaviors in material science, just to cite a few; see [5], [13] for more details on applications of PDEs. Due to the relevance of PDEs in engineering applications, over the last two decades, and further back, control of PDEs has attracted the interest of researchers and a large number of tools has been developed for the analysis and the design of control systems governed by PDEs; see [13] and the references therein. One of the unique challenges in the control of PDEs is that in the majority of the applications, actuators and sensors are located only at the boundaries of the PDE. In this case, one talks about boundary control [13]. Obviously, the design of boundary feedback controllers is a hard problem. Indeed, loosely speaking, boundary control can be somehow compared to a form of underactuation in which the controller has limited authority on the system due to boundary actuation; let alone the fact that boundary sensing allows one to gather only limited information on the system state. Different methodologies to design boundary feedback controllers for PDEs exist; e.g., Lyapunov methods [5], [20], backstepping transformation [13], or frequency domain approaches as in [15]. More recently, the use of optimization tools commonly used for the analysis and the design of control systems governed by ordinary differential equations has been explored in the context of PDEs; see [21], [17], [4].

Francesco Ferrante and Christophe Prieur are with Univ. Grenoble Alpes, CNRS, Grenoble INP, GIPSA-lab, 38000 Grenoble, France. Email: \{francesco.ferrante, christophe.prieur\}@ gipsa-lab.fr.

Research by F. Ferrante has been partially supported by the CNRS-INS2I under the JCJC grant CoBrA and by the Grenoble Institute of Technology under the grant CrYStAL.
In this paper, we focus on linear one dimensional conservation laws, which are a specific class of hyperbolic PDEs. The main interest in hyperbolic PDEs is that such equations are ubiquitous in physical applications; see [5] for a recent monograph on hyperbolic PDEs. Recently, boundary control of hyperbolic PDEs has seen a growing interest in the community; see [7], [14], [20], just to cite a few. In particular, in [7], dynamic output feedback stabilization of linear hyperbolic PDEs has been considered. In [14], the authors analyzed the use of switching rules for the stabilization of system of linear conservation laws. In [20] the problem of designing integral controllers for nonlinear systems governed by scalar hyperbolic partial differential equations is studied.

Motivated by the recent research efforts in the boundary control of hyperbolic PDEs, in this article we address the problem of designing a boundary feedback controller for a system on $n 1-\mathrm{D}$ linear conservation laws in the presence of measurement noise. More specifically, we propose a systematic approach for the design of static boundary controllers to achieve: closed-loop asymptotic stability and robustness with quantifiable margins with respect to energy bounded measurement noise. To achieve this goal, first we provide Lyapunov-like sufficient conditions in the form of functional inequalities to ensure a bound on the spatial norm of the closed-loop system state that depends on the energy of the measurement noise. These inequalities generalize previous results for ordinary differential equations; cf. [3]. Then, by operating a particular selection for the Lyapunov functional, we turn the considered control design problem into the feasibility problem of some matrix inequalities. The proposed conditions are embedded into a convex optimization setup to allow for the design of controllers achieving measurement noise rejection.

The remainder of the paper is organized as follows. Section II presents the system under consideration and illustrates the problem we solve. Section III is devoted to the main results of our paper. Finally, Section IV showcases the application of our methodology in a numerical example. Due to space constraints, proofs of the main results are omitted and will be published elsewhere.

\section{A. Notation}

The set $\mathbb{R}_{\geq 0}$ represents the set of nonnegative real scalars, $\mathbb{R}^{n \times m}$ denotes the set of the $n \times m$ real matrices, $\mathbb{S}_{p}^{n}$ is the set of real symmetric positive definite matrices of dimension $n$, and $\mathbb{D}_{p}^{n}$ is the set of real diagonal positive definite matrices of 
dimension $n$. In partitioned symmetric matrices, the symbol - stands for symmetric blocks. The symbol I denotes the identity matrix. Given two matrices $A_{1}$ and $A_{2}$, we denote by $A_{1} \oplus A_{2}$ the block diagonal matrix $\left[\begin{array}{cc}A_{1} & \mathbf{0} \\ \mathbf{0} & A_{2}\end{array}\right]$. The identity matrix is denoted by $\mathbf{I}$, whereas the null matrix is denoted by 0. For a matrix $A \in \mathbb{R}^{n \times m}, A^{\top}$ denotes the transpose of $A$, and $\operatorname{He}(A)=A+A^{\top}$. For a symmetric matrix, $A$, positive definiteness (negative definiteness) and positive semidefiniteness (negative semidefiniteness) are denoted, respectively, by $A \succ 0(A \prec 0)$ and $A \succeq 0(A \preceq 0)$. Let $X \subset \mathbb{R}^{n}, Y \subset \mathbb{R}$, $x \in X$, and $f: X \rightarrow Y$, the symbol $\nabla f(x)$ denotes the gradient of $f$ at $x$. For a vector $x \in \mathbb{R}^{n},|x|$ denotes its Euclidean norm. Given $x, y \in \mathbb{R}^{n}$, we denote by $\langle x, y\rangle_{\mathbb{R}^{n}}$ the standard Euclidean inner product. Let $X$ and $Y$ be linear normed spaces, the symbol $\mathscr{L}(X, Y)$ denotes the space of all bounded linear operators from $X$ to $Y$. Let $U \subset \mathbb{R}$ and $V \subset$ $\mathbb{R}^{n}$, and $f: U \rightarrow V$. We denote by $\|f\|_{\mathcal{L}^{\infty}}=\sup _{x \in U}|f(x)|$ and $\|f\|_{\mathcal{L}^{2}}=\left(\int_{U}|f(x)|^{2} d x\right)^{\frac{1}{2}}$, respectively, the uniform norm and the $\mathcal{L}^{2}$ norm of $f$. Let $U \subset \mathbb{R}$ and $V \subset \mathbb{R}^{n}$. The symbols $\mathcal{C}^{k}(U ; V)$ and $\mathcal{H}^{k}(U ; V)$ denote, respectively, the sets of class- $k$ functions $f: U \rightarrow V$ and of the set of functions $f: U \rightarrow V$ such that $f \in \mathcal{L}^{2}(U ; V)$ and for each $j=1,2, \ldots, k, \partial^{j} f \in \mathcal{L}^{2}(U ; V)$, where $\partial^{j} f$ stands for the weak derivative of order $j$ of $f$. Given $f: U \subset \mathbb{R} \rightarrow V$, we say that $f \in \mathcal{L}^{2}$ if $\|f\|_{\mathcal{L}^{2}}$ is finite.

Definition 1 ([9]). Let $X$ and $Y$ be linear normed spaces, $U$ be an open subset of $X, f: U \rightarrow Y$, and $x \in U$. We say that $f$ is Fréchet differentiable at $x$ if there exists $L \in \mathscr{L}(X, Y)$ such that

$$
\lim _{h \rightarrow 0} \frac{\|f(x+h)-f(x)-L h\|_{Y}}{\|h\|_{X}}=0
$$

In particular $L$ is the Fréchet derivative of $f$ at $x$ and is denoted by $D f(x)$. When $X=\mathbb{R}$, with a slight abuse of notation, we denote

$$
D f(x)=\lim _{h \rightarrow 0} \frac{f(x+h)-f(x)}{h}
$$

\section{Problem Statement}

We consider boundary feedback control of a system of $n$ linear 1-D conservation laws written formally as:

$$
\begin{array}{ll}
\partial_{t} x(t, z)+\Lambda \partial_{z} x(t, z)=0 & \forall(t, z) \in \mathbb{R}_{\geq 0} \times[0,1] \\
x(t, 0)=H x(t, 1)+B u(t) & \forall t \in \mathbb{R}_{\geq 0}
\end{array}
$$

where $\partial_{t} x$ and $\partial_{z} x$ denote, respectively, the derivative of $x$ with respect to "time" and with respect to the "spatial" variable $z,[0,1] \ni z \mapsto x(\cdot, z) \in \mathbb{R}^{n}$ is the system state, $u \in \mathbb{R}^{n}$ is the control input, and $\Lambda \in \mathbb{D}_{p}^{n}, H \in \mathbb{R}^{n \times n}$, and $B \in \mathbb{R}^{n \times n}$ are given matrices. In this paper, we consider the following standing assumption:

Assumption 1. The input matrix $B$ is nonsingular.

Remark 1. In this paper, we assume that the number of control inputs of system is equal to $n$, that is the number of scalar conservation laws describing (1). In other words, we assume that each conservation law in (1) can be controlled independently; this assumption is verified in many cases of practical interest; see [5]. As such, assuming that B is nonsingular is a reasonable assumption in this context. The extension of the results in this paper to the case of conservation laws with $m<n$ inputs is currently part of our work.

In particular, (1) takes the form of a system of $n$ 1D boundary-controlled linear hyperbolic PDEs, for which several fundamental results can be found in [5].

Remark 2. Assuming that $\Lambda$ is positive definite implies that system (1) is characterized by positive convecting speeds. However, such an assumption does not add any loss of generality. Indeed, any system of 1-D linear conservation laws can be expressed as in (1) via an invertible change of variables; e.g., [8], [19].

We further assume that the state $x(\cdot, z)$ is measurable only at the boundary point $z=1$. Specifically, for all $t \geq 0$, the measurable output of the system reads as

$$
y(t)=x(t, 1)+d(t)
$$

where $d \in \mathcal{L}^{2}\left(\mathbb{R}_{\geq 0}, \mathbb{R}^{n}\right)$ represents an energy-bounded measurement noise.

Our goal is to design a static feedback control law $u$, which stabilizes system (1) in some appropriate sense and with quantifiable robustness margins with respect to the measurement noise $d$. More precisely, in this paper we focus on static output feedback laws of the form $y \mapsto u(y)=K y$, where $K \in \mathbb{R}^{n \times n}$ is the control gain that needs to be designed. By setting $H_{c l}:=H+B K$, the closed-loop system can be formally written as:

$$
\begin{array}{ll}
\partial_{t} x(t, z)+\Lambda \partial_{z} x(t, z)=0 & \forall(t, z) \in \mathbb{R}_{\geq 0} \times[0,1] \\
x(t, 0)=H_{c l} x(t, 1)+B K d(t) & \forall t \geq 0
\end{array}
$$

see Fig. 1.

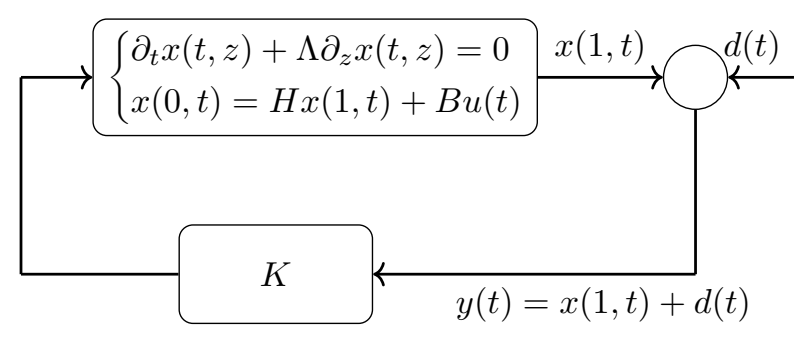

Fig. 1: Closed-loop system.

A. Discussion on the existence and notion of the solutions to the closed-loop system

Similarly as in [12], in this paper, we focus on solutions to (1) that are sufficiently regular. In particular, the following notion of solutions is considered throughout the paper. 
Definition 2 (Strong solution). Let $d \in \mathcal{C}^{0}\left(\mathbb{R}_{\geq 0} ; \mathbb{R}^{n}\right)$ and $\varphi \in \mathcal{C}^{1}\left(\mathbb{R}_{\geq 0} \times[0,1] ; \mathbb{R}^{n}\right)$. We say that $\varphi$ is a strong solution to (1) with input $d$ if for all $(t, z) \in \mathbb{R}_{>0} \times(0,1)$

$$
\begin{aligned}
& \partial_{t} \varphi(t, z)+\Lambda \partial_{z} \varphi(t, z)=0 \\
& \varphi(t, 0)=H_{c l} \varphi(t, 1)+B K d(t)
\end{aligned}
$$

Noting that system (3) is linear. Therefore, the characteristic curves are straight lines and, under some compatibility conditions between the boundary condition and the initial condition in (3), existence and uniqueness of strong solutions can be ensured. In particular, one has the following result.

Proposition 1. Let $x_{0} \in \mathcal{C}^{1}\left([0,1] ; \mathbb{R}^{n}\right)$ and $d \in$ $\mathcal{C}^{1}\left(\mathbb{R}_{\geq 0} ; \mathbb{R}^{n}\right)$ such that the following compatibility conditions are verified

$$
\begin{aligned}
& x_{0}(0)=H_{c l} x_{0}(1)+B K d(0) \\
& -\Lambda x_{0}(0)=-H_{c l} \Lambda x_{0}(1)+B K \frac{d}{d t} d(0)
\end{aligned}
$$

Then, there exists a unique strong solution $\phi \in \mathcal{C}^{1}\left(\mathbb{R}_{\geq 0} \times\right.$ $\left.[0,1] ; \mathbb{R}^{n}\right)$ with input $d$ to (3) such that $x(0, \cdot)=x_{0}$.

The proof of the above result can be easily constructed by building upon the rationals in [5, Page 57] and it is therefore omitted.

In the light of the above result, given any $d \in$ $\mathcal{C}^{1}\left(\mathbb{R}_{\geq 0} ; \mathbb{R}^{n}\right)$, we define

$$
\begin{aligned}
\mathcal{X}_{d}:= & \left\{x_{0} \in \mathcal{C}^{1}\left([0,1] ; \mathbb{R}^{n}\right):\right. \\
& x_{0}(0)=H_{c l} x_{0}(1)+B K d(0), \\
& \left.-\Lambda x_{0}(0)=-H_{c l} \Lambda x_{0}(1)+B K \frac{d}{d t} d(0)\right\}
\end{aligned}
$$

Moreover, given $d \in \mathcal{C}^{1}\left(\mathbb{R}_{\geq 0} ; \mathbb{R}^{n}\right)$ and any $x_{0} \in \mathcal{X}_{d}$, throughout the paper we denote the unique strong solution to (3) from $x_{0}$ by $(t, z) \mapsto \varphi\left(t, z, x_{0}, d\right)$.

Now we are in a position to formally state the problem we solve in this paper.

Problem 1. Given $H \in \mathbb{R}^{n \times n}, B \in \mathbb{R}^{n \times m}$, and $\Lambda \in \mathbb{D}_{p}^{n}$. Design a control gain $K \in \mathbb{R}^{n \times n}$ and determine $\kappa, \omega, \gamma \in$ $\mathbb{R}_{>0}$ such that for each $d \in \mathcal{L}^{2}\left(\mathbb{R}_{\geq 0} ; \mathbb{R}^{n}\right) \cap \mathcal{C}^{1}\left(\mathbb{R}_{\geq 0} ; \mathbb{R}^{n}\right)$ and any $x_{0} \in \mathcal{X}_{d}$ one has, for all $t \in \mathbb{R}_{\geq 0}$

$$
\left\|\varphi\left(t, \cdot, x_{0}, d\right)\right\|_{\mathcal{L}^{2}} \leq \kappa e^{-\omega t}\left\|x_{0}\right\|_{\mathcal{L}^{2}}+\gamma\|d\|_{\mathcal{L}^{2}}
$$

\section{MAIN RESUlts}

In this section we propose sufficient conditions for the solution to Problem 1 in the form of functional inequalities. To this end, we first provide an alternative characterization of strong solutions to (3) via the definition of an abstract differential equation in a subset of $\mathcal{H}^{1}\left([0,1] ; \mathbb{R}^{n}\right)$. The definition of such a differential equation allows one to establish a linear relationship between $\partial_{z} x$ and $\partial_{t} x$ for any strong solution.
For each $d \in \mathbb{R}^{m}$ define $\mathcal{A}_{d}: \operatorname{dom} \mathcal{A}_{d} \rightarrow \mathcal{H}^{1}\left([0,1] ; \mathbb{R}^{n}\right)$ with

$$
\begin{aligned}
\operatorname{dom} \mathcal{A}_{d}:=\left\{w \in \mathcal{H}^{1}\left([0,1] ; \mathbb{R}^{n}\right):\right. & \\
& w(0)=(H+B K) w(1)+B K d\} \\
\mathcal{A}_{d} w:= & -\Lambda \frac{d}{d z} w(z)
\end{aligned}
$$

Then, the closed-loop system can be formally rewritten as the following abstract differential equation

$$
\dot{w}=\mathcal{A}_{d} w
$$

It can be easily shown that given $d \in \mathcal{C}^{1}\left(\mathbb{R}_{\geq 0} ; \mathbb{R}^{n}\right)$ and $x_{0} \in \mathcal{X}_{d}$, the function $t \mapsto \phi(t):=\varphi\left(t, \cdot, x_{0}, d\right)$ is such that for all $t \in \mathbb{R}_{\geq 0}$ one has

$$
\begin{aligned}
& \phi(t) \in \operatorname{dom} \mathcal{A}_{d(t)} \\
& D \phi(t)=\mathcal{A}_{d(t)} \phi(t)
\end{aligned}
$$

where $D \phi$ denotes the Fréchet derivative of $\phi$ with respect to $t$.

\section{A. Sufficient Conditions}

Theorem 1. Let $\alpha: \mathcal{C}^{1}\left([0,1] ; \mathbb{R}^{n}\right) \rightarrow \mathbb{R}_{\geq 0}$ and $\mathcal{U} \supset$ $\mathcal{C}^{1}\left([0,1] ; \mathbb{R}^{n}\right)$ be a linear normed space. Assume that there exist a Fréchet differentiable functional $V: \mathcal{U} \rightarrow \mathbb{R}_{\geq 0}$ and $c_{1}, c_{2}, c_{3}, \chi \in \mathbb{R}_{>0}$ such that for each $d \in \mathbb{R}^{m}$ and $w \in$ $\operatorname{dom} \mathcal{A}_{d}$

$$
\begin{array}{r}
c_{1} \alpha(w)^{2} \leq V(w) \leq c_{2} \alpha(w)^{2} \\
D V(w) \circ \mathcal{A}_{d} w \leq-c_{3} V(w)+\chi^{2}|d|^{2}
\end{array}
$$

Let $d \in \mathcal{L}^{2}\left(\mathbb{R}_{\geq 0} ; \mathbb{R}^{n}\right) \cap \mathcal{C}^{1}\left(\mathbb{R}_{\geq 0} ; \mathbb{R}^{n}\right), x_{0} \in \mathcal{X}_{d}$, and for each $t \in \mathbb{R}_{\geq 0}, \phi(t):=\varphi\left(t, \cdot, x_{0}, \bar{d}\right)$. Then, for all $t \in \mathbb{R}_{\geq 0}$, one has

$$
\alpha(\phi(t)) \leq e^{-\frac{c_{3}}{2} t}\left[\frac{c_{2}}{c_{1}}\right]^{\frac{1}{2}} \alpha\left(x_{0}\right)+\frac{\chi}{\sqrt{c_{1}}}\|d\|_{\mathcal{L}^{2}}
$$

Inequality (9) can be seen as a special form of dissipation inequality for the abstract differential equation (6). Indeed, the functionals $w \mapsto V(w)$ and $(w, d) \mapsto-c_{3} V(w)+\chi^{2} d^{\top} d$ can be thought, respectively, as a storage function and a supply rate; see [10], [22]. Dissipation inequalities for systems governed by PDEs have been already considered in [1].

Remark 3. By relying on the representation of the closedloop system given by the abstract differential equation (6), we are able to formulate a dissipation inequality-like condition in the form of a functional inequality in a subspace of $\mathcal{H}^{1}\left([0,1] ; \mathbb{R}^{n}\right) \times \mathbb{R}^{n}$. This provides an elegant generalization to PDEs of the well-known dissipation inequalities for ordinary differential equations; see, e.g., [3]. It is interesting to observe that the gradient of $V$ is replaced in (9) by the Fréchet derivative and these two objects coincide in finite dimensional spaces.

Theorem 1 provides sufficient conditions for the solution to Problem 1. However, the applicability of such a result 
requires the construction of the (storage) functional $V$ and this is a challenging task in general. In [1], the authors propose a constructive approach based on optimization techniques for the construction of storage functionals for a wide class of PDEs. Although the results in [1] cover a large class of PDEs, their applicability to the analysis of the closed-loop system (3) does not seem straightforward due to different boundary conditions. Therefore, with the objective of deriving a systematic design algorithm tailored to linear conservations laws, in the following section we propose a specific structure for the functional $V$ in Theorem 1, which allows one to cast the solution to Problem 1 in the solution to some matrix inequalities.

\section{B. Construction of the functional $V$}

The result given next provides sufficient conditions for Theorem 1 to hold in the form of a matrix inequality. The result builds on the use of the following functional

$$
V(X)=\int_{0}^{1} e^{-\mu z} X(z)^{\top} P X(z) d z
$$

Theorem 2. If there exists $P \in \mathbb{D}_{p}^{n}, K \in \mathbb{R}^{n \times n}, \mu, \chi \in \mathbb{R}_{>0}$ such that

$$
\left[\begin{array}{cc}
(H+B K)^{\top} \Lambda P(H+B K)-e^{-\mu} \Lambda P & (H+B K)^{\top} \Lambda P B K \\
\bullet & K^{\top} B^{\top} \Lambda P B K-\chi^{2} \mathbf{I}
\end{array}\right] \prec 0
$$

Then, $K$ solves Problem 1 and in particular (4) holds with

$$
\begin{aligned}
& \omega=\frac{1}{2} \mu \lambda_{\min }(\Lambda), \quad \kappa=\sqrt{\frac{\lambda_{\max }(P)}{\lambda_{\min }(P)}} e^{\frac{\mu}{2}} \\
& \gamma=\frac{\chi}{\sqrt{\lambda_{\min }(P)}} e^{\frac{\mu}{2}}
\end{aligned}
$$

Remark 4. Although our approach is conservative, (12) seems to suggest that disturbance rejection and convergence speed are antagonistic objectives. Indeed, higher values of $\mu$ are more likely to lead to poor robustness with respect to boundary disturbances, but that potentially allows one to achieve faster transient response. This aspect will be illustrated in a numerical example in Section IV.

Theorem 2 recasts the solution to Problem 1 as the feasibility problem of a matrix inequality, i.e., (11) . However, due to its form, such a condition is not computationally tractable to obtain a solution to Problem 1. Indeed, condition (11) is nonlinear in the variables $P, K, \chi$, and $\mu$, thus it is hardly tractable from a numerical standpoint [6]. Although the nonlinearities involving the variables $\chi$ and $\mu$ can be easily addressed, further work is needed to tackle the trilinear terms involving $P$ and $K$. This aspect is considered in the subsequent section.

\section{Control Design}

Theorem 3. Let $P \in \mathbb{D}_{p}^{n}, \mu, \chi \in \mathbb{R}_{>0}$, and $K \in \mathbb{R}^{n \times n}$ be given. The following two statements are equivalent

(a) (11) holds; (b) There exists $Q \in \mathbb{R}^{n \times n}$ such that

$$
\left[\begin{array}{ccc}
\operatorname{He}(Q)+\Lambda P & -Q^{\top}(H+B K) & -Q^{\top} B K \\
\bullet & -e^{-\mu} \Lambda P & 0 \\
\bullet & \bullet & -\chi^{2} \mathbf{I}
\end{array}\right] \prec 0
$$

The above result provides an equivalent condition to (11) in which no trilinear terms appear. The result given next shows that a simple change of variables turns (13) into a linear matrix inequality, modulo the nonlinearity involving the variable $\mu$.

Corollary 1. If there exist $P \in \mathbb{D}_{p}^{n}, \mu, \theta \in \mathbb{R}_{>0}, Q \in \mathbb{R}^{n \times n}$, and $Y \in \mathbb{R}^{n \times n}$ such that ${ }^{1}$

$$
\left[\begin{array}{ccc}
\operatorname{He}(Q)+\Lambda P & -Q^{\top} H-Y & -Y \\
\bullet & -e^{-\mu} \Lambda P & 0 \\
\bullet & \bullet & -\theta \mathbf{I}
\end{array}\right] \prec 0
$$

Then, $Q$ is nonsingular and $K=B^{-1} Q^{-\top} Y$ solves Problem 1. In particular, (4) holds with $\omega$ and $\kappa$ defined as in (12) and

$$
\gamma=\sqrt{\frac{\theta}{\lambda_{\min }(P)}} e^{\frac{\mu}{2}}
$$

\section{Optimization Aspects}

In the formulation of Problem 1, no specific requirements on the scalar $\gamma$ are considered. On the other hand, it is obvious that to reduce the effect of the measurement noise on the closed-loop system, the controller gain $K$ should be designed so that (4) holds with a minimal $\gamma$. This goal can be achieved by considering the following optimization problem

$$
\begin{array}{ll}
\inf _{P, Y, \mu, Q, \theta} & \frac{\theta}{\lambda_{\min }(P)} e^{\frac{\mu}{2}} \\
\text { subject to } & (14), P \in \mathbb{D}_{p}^{n}, \mu>0, \theta>0
\end{array}
$$

At this stage it is worthwhile to remark that the solution to the above optimization problem is in general challenging due to (14) being nonlinear in the decision variable $\mu$ and the objective function being nonlinear in the decision variables $\theta$ and $P$. With the aim of obtaining a computationally affordable design algorithm, we want to tackle the solution to (15) via semidefinite programming tools [6]. While the nonlinearity involving the variable $\mu$ can be easily overcome by performing a line search, the objective function needs to be suitably reformulated to get a linear objective. To this end, let $c \in \mathbb{R}_{>0}$, consider the following additional constraint

$$
\left[\begin{array}{cc}
P & \mathbf{I} \\
\bullet & c \mathbf{I}
\end{array}\right] \succeq 0
$$

which holds if and only if $c^{-1} \leq \lambda_{\min }(P)$. The satisfaction of (16) ensures that

$$
\frac{\theta}{\lambda_{\min }(P)} \leq \theta c
$$

\footnotetext{
${ }^{1}$ There is a typo in equation (14) in the published proceedings, i.e., $H^{\top}$ should be $H$.
} 
Then, since $\mathbb{R}_{>0} \times \mathbb{R}_{>0} \ni(\theta, c) \mapsto \theta c$ and $\mathbb{R}_{>0} \times \mathbb{R}_{>0} \ni$ $(\theta, c) \mapsto \theta+c$ are monotonically related each other, one can consider the following optimization problem

$$
\begin{array}{ll}
\inf _{P, \mu, Q, Y, \theta} & (\theta+c) e^{\frac{\mu}{2}} \\
\text { subject to } & (16), P \in \mathbb{D}_{p}^{n}, \mu>0, \theta>0, c>0 \\
& {\left[\begin{array}{ccc}
\operatorname{He}(Q)+\Lambda P & -Q^{\top} H^{\top}-Y & -Y \\
\bullet & -e^{-\mu} \Lambda P & 0 \\
\bullet & \bullet & -\theta \mathbf{I}
\end{array}\right] \prec 0}
\end{array}
$$

When $\mu$ is selected, optimization problem (17) is a semidefinite program, i.e., an optimization problem over linear matrix inequality constraints with linear objective. Hence, it can be efficiently solved in polynomial time via numerical available solvers; see, e.g., [6].

Remark 5. Although the main focus of this paper is on control design, it is worthwhile to observe that the results we propose can be used as an analysis tool for a given control gain. In particular, given a control gain $K$, an optimization problem analogous to (17) can be formulated to certify the existence of a bound (4), while reducing the pessimism in the estimation of the gain $\gamma$.

\section{NUMERICAL EXAMPLE}

Consider the example in [11] in which system (1) is defined by the following data

$$
\Lambda=\left[\begin{array}{cc}
1 & 0 \\
0 & \sqrt{2}
\end{array}\right], H=\left[\begin{array}{cc}
0 & 1.1 \\
1 & 0
\end{array}\right], B=\mathbf{I}
$$

We consider the solution to Problem 1 via the solution to the optimization problem (17), which can be effectively obtained by performing a line search on $\mu$. In particular, for each $\mu>0$, let $P_{\mu}^{\star}, \theta_{\mu}^{\star}, Q_{\mu}^{\star}$ be the solution to the optimization problem $^{2}$ (17). In Fig. 2 we report the function

$$
\mu \mapsto \gamma^{\star}(\mu)=\frac{\theta_{\mu}^{\star}}{\sqrt{\lambda_{\min }\left(P_{\mu}^{\star}\right)}} e^{\frac{\mu}{2}}
$$

which, for each $\mu$, provides the smallest achievable value of $\gamma$ for which the bound in (4) holds for the closed-loop system when the control gain $K$ is designed as in Corollary 1. The picture suggests that $\mu \mapsto \gamma^{\star}(\mu)$ is increasing. This fact aligns with the discussion in Remark 4 and emphasizes that a tradeoff between convergence speed and disturbance rejection may be needed. To get an insight on the influence of the value of $\mu$ on the closed-loop behavior, we compare the behavior of the closed-loop system obtained via different selections of the parameter $\mu$. In particular, we consider $\mu=\mu_{1}:=0.01$ and $\mu=\mu_{2}:=0.5$. By solving optimization problem (17) with $\mu=\mu_{1}$ and $\mu=\mu_{2}$, one gets, respectively,

\footnotetext{
${ }^{2}$ Numerical solutions to SDP problems are obtained in Matlab ${ }^{\circledR}$ via Mosek [2] thanks to YALMIP [16]. Numerical integration of hyperbolic PDEs is performed via the use of the Lax-Friedrichs (Shampine's twostep variant) scheme implemented in Matlab ${ }^{\circledR}$ by Shampine [18]. Code at https://github.com/f-ferrante/HyperbolicExl
}

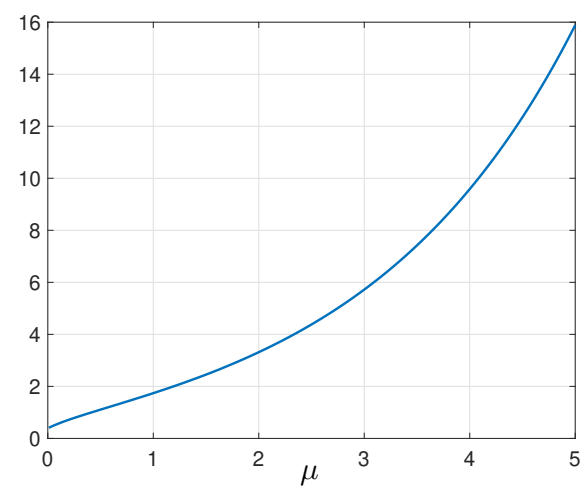

Fig. 2: The function $\mu \mapsto \gamma^{\star}(\mu)$.

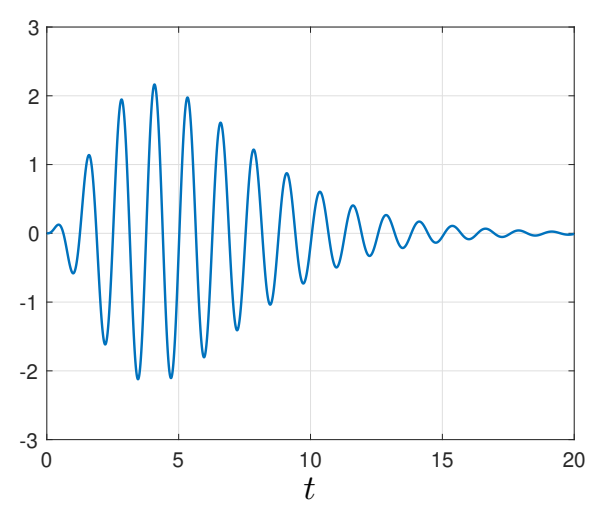

Fig. 3: The function $t \mapsto \widehat{d}(t)$ in (18).

the following results

$$
\begin{array}{ll}
K_{1} \approx\left[\begin{array}{cc}
0 & -0.1155 \\
-0.095 & 0
\end{array}\right] & \gamma_{1} \approx 0.3863 \\
K_{2} \approx\left[\begin{array}{cc}
0 & -0.5242 \\
-0.4214 & 0
\end{array}\right] & \gamma_{2} \approx 1.0397
\end{array}
$$

Moreover, to evaluate the effectiveness of our design strategy, we consider the following stabilizing gain for the closed-loop system given in [11]

$$
K_{3}=\left[\begin{array}{cc}
0 & -0.7 \\
-1 & 0
\end{array}\right]
$$

Consider the following disturbance; see Fig. 3

$$
t \mapsto d(t):=\left[\begin{array}{l}
1 \\
1
\end{array}\right] t^{2} \sin (5 t) e^{-\frac{1}{2} t}:=\left[\begin{array}{l}
1 \\
1
\end{array}\right] \widehat{d}(t)
$$

and observe that $d \in \mathcal{L}^{2}\left(\mathbb{R}_{\geq 0} ; \mathbb{R}^{n}\right) \cap \mathcal{C}^{1}\left(\mathbb{R}_{\geq 0} ; \mathbb{R}^{n}\right)$ the null function belongs to $\mathcal{X}_{d}$. In Fig. 4, we compare the evolution of the $\mathcal{L}^{2}$-norm of the closed-loop system state from zero initial conditions in response to the disturbance (18) obtained for the three control gains. As expected, the gain leading to a smaller value of $\gamma$, i.e., $K_{1}$ provides better disturbance rejection.

To better assess the tradeoff between convergence speed and disturbance rejection, in Fig. 5 we compare the evolution 
of the $\mathcal{L}^{2}$-norm of the closed-loop system, with the three considered gains, in response to the disturbance (18) from the following initial condition

$$
[0,1] \ni z \mapsto x_{0}(z):=\left[\begin{array}{l}
\cos (4 \pi z)-1 \\
\cos (2 \pi z)-1
\end{array}\right]
$$

Observe that, with $d$ as in (18), $x_{0} \in \mathcal{X}_{d}$. Fig. 5 clearly points

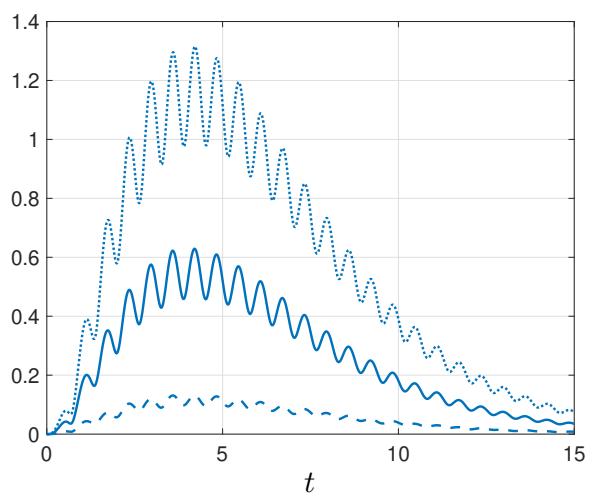

Fig. 4: The evolution of $t \mapsto\|\varphi(t, \cdot, 0, d)\|_{\mathcal{L}^{2}}$, with $d$ selected as in (18), for different control gains: $K_{1}$ (dashed-line), $K_{2}$ (solid-line), $K_{3}$ (dotted-line).

out that overall the best performance is achieved with the gain $K_{2}$ for which a tradeoff between disturbance rejection and convergence speed has been considered.

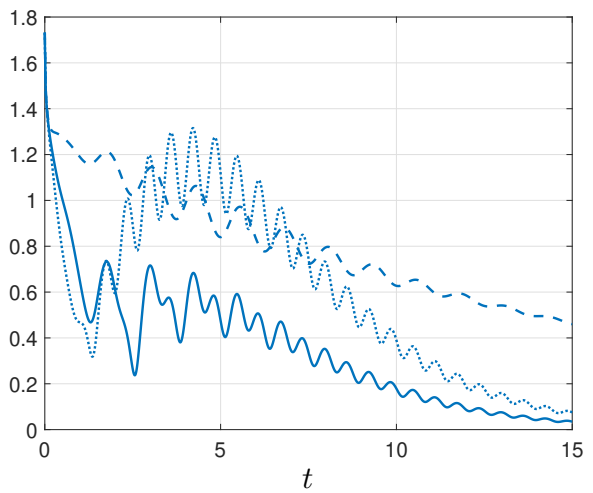

Fig. 5: The evolution of $t \mapsto\left\|\varphi\left(t, \cdot, x_{0}, d\right)\right\|_{\mathcal{L}^{2}}$, with $d$ and $x_{0}$ selected, respectively, as in (18) and (19), for different control gains: $K_{1}$ (dashed-line), $K_{2}$ (solid-line), $K_{3}$ (dottedline).

\section{CONCLUSiON}

In this paper, we considered the problem of designing a static boundary controller to stabilize a system governed by a $n$ linear conservation laws, in the presence of energybounded measurement noise. The controller is designed to induce a bound on the $\mathcal{L}^{2}$ norm of the state. Such a bound is exponentially converging in the $\mathcal{L}^{2}$ norm of the initial condition and quadratic in the $\mathcal{L}^{2}$ norm of the measurement noise. By pursuing a Lyapunov approach, the control design problem is recast into the feasibility problem of some matrix inequalities. Semidefinite programming tools are used to design the controller while achieving a tradeoff between convergence speed and disturbance rejection. Numerical simulations illustrate the effectiveness of the proposed control design strategy in an example.

\section{REFERENCES}

[1] M. Ahmadi, G. Valmorbida, and A. Papachristodoulou. Dissipation inequalities for the analysis of a class of pdes. Automatica, 66:163171, 2016.

[2] MOSEK ApS. The MOSEK optimization toolbox for MATLAB manual. Version 7.1 (Revision 28)., 2015.

[3] M. Arcak, D. Angeli, and E. Sontag. A unifying integral iss framework for stability of nonlinear cascades. SIAM Journal on Control and Optimization, 40(6):1888-1904, 2002.

[4] M. Barreau, A. Seuret, F. Gouaisbaut, and L. Baudouin. Lyapunov stability analysis of a string equation coupled with an ordinary differential system. To appear in the IEEE Transactions on Automatic Control, 2018.

[5] G. Bastin and J.-M. Coron. Stability and Boundary Stabilization of 1-D Hyperbolic Systems, volume 88 of Progress in Nonlinear Differential Equations and Their Applications. Springer, 2016.

[6] S. Boyd, L. El Ghaoui, E. Feron, and V. Balakrishnan. Linear Matrix Inequalities in System and Control Theory. Society for Industrial and Applied Mathematics, June 1997.

[7] F. Castillo, E. Witrant, C. Prieur, and L. Dugard. Dynamic boundary stabilization of linear and quasi-linear hyperbolic systems. In Proceedings of the IEEE 51st Conference on Decision and Control, pages 2952-2957, 2012.

[8] F. Castillo, E. Witrant, C. Prieur, and L. Dugard. Boundary observers for linear and quasi-linear hyperbolic systems with application to flow control. Automatica, 49(11):3180-3188, 2013.

[9] W. Cheney. Analysis for applied mathematics, volume 208. Springer Science \& Business Media, 2013.

[10] C. Ebenbauer, T. Raff, and F. Allgöwer. Dissipation inequalities in systems theory: An introduction and recent results. In Invited lectures of the international congress on industrial and applied mathematics, volume 2007, pages 23-42, 2009.

[11] N. Espitia, A. Tanwani, and S. Tarbouriech. Stabilization of boundary controlled hyperbolic PDEs via Lyapunov-based event triggered sampling and quantization. In Proceedings of the IEEE 56th Annual Conference on Decision and Control, pages 1266-1271. IEEE, 2017.

[12] I. Karafyllis and M. Krstic. ISS with respect to boundary disturbances for 1-D parabolic PDEs. IEEE Transactions on Automatic Control, 61(12):3712-3724, 2016

[13] M. Krstic and A. Smyshlyaev. Boundary Control of PDEs: A Course on Backstepping Designs. SIAM, Philadelphia, PA, USA, 2008.

[14] P.-O. Lamare, A. Girard, and C. Prieur. An optimisation approach for stability analysis and controller synthesis of linear hyperbolic systems. ESAIM: Control, Optimisation and Calculus of Variations, 22(4):1236-1263, 2016.

[15] X. Litrico and V. Fromion. Modeling and control of hydrosystems. Springer Science \& Business Media, 2009.

[16] J. Lofberg. Yalmip: A toolbox for modeling and optimization in matlab. In Computer Aided Control Systems Design, 2004 IEEE International Symposium on, pages 284-289. IEEE, 2004.

[17] M. Safi, L. Baudouin, and A. Seuret. Tractable sufficient stability conditions for a system coupling linear transport and differential equations. Systems \& Control Letters, 110:1-8, 2017.

[18] L. F. Shampine. Solving hyperbolic pdes in matlab. Applied Numerical Analysis \& Computational Mathematics, 2(3):346-358, 2005.

[19] Y. Tang, C. Prieur, and A. Girard. Tikhonov theorem for linear hyperbolic systems. Automatica, 57:1-10, 2015.

[20] N.-T. Trinh, V. Andrieu, and C.-Z. Xu. Design of integral controllers for nonlinear systems governed by scalar hyperbolic partial differential equations. IEEE Transactions on Automatic Control, 62(9):4527-4536, 2017.

[21] G. Valmorbida, M. Ahmadi, and A. Papachristodoulou. Stability analysis for a class of partial differential equations via semidefinite programming. IEEE Transactions on Automatic Control, 61(6):16491654,2016

[22] J. C. Willems. Mechanisms for the stability and instability in feedback systems. Proceedings of the IEEE, 64(1):24-35, 1976. 